\title{
Orbital floor fracture repair with implants: a retrospective study
}

\author{
Yong Jig Lee \\ Department of Plastic and \\ Reconstructive Surgery, Daegu Catholic \\ University School of Medicine, Daegu, \\ Korea
}

Background: Although prompt surgery after an orbital fracture is preferable, the actual timing of surgery in real-world settings varies. Therefore, this study investigated the outcomes of implant surgery for inferior orbital wall fractures by comparing three groups according to the time interval between the injury and surgery.

Methods: A retrospective review was conducted of patients' medical charts and initial computed tomography images from 2009 to 2020. The time to treatment was chosen by patients or their guardians based on the patients' comorbidities and the physician's explanation. The patients were divided into three groups according to the time of surgery (group 1:3-7 days, group 2: 8-14 days, group 3: 15 or more days). Data were collected on age, the time interval until surgery, the dimensions of the defect, the operation time, the follow-up period, and the postoperative paresthesia score (ranging from 0 to 10). The outcomes were evaluated using a 4-point scale: $4=$ good (no complications), 3 =fair (no subjective symptoms), 2 =poor (remaining paresthesia), and 1=very poor (strabismus and/or enophthalmos).

Results: The study included 85 patients with unilateral fractures who underwent surgery from 3 to 93 days after injury. The overall score distribution of the surgical outcomes was as follows: good $=63$, fair $=7$, poor $=6$, and very poor $=9$. The three groups showed no significant differences in the transverse dimension of the injury $(p=0.110)$ or the anteroposterior dimension $(p=0.144)$. In groups 1,2 , and 3 , the postoperative outcome scores were $3.84 \pm 0.37,3.63 \pm 0.87$, and $2.93 \pm 1.33$ $(p=0.083)$, and the percentage of patients with good outcomes was $84 \%, 81.25 \%$, and $57.14 \%$, respectively.

Conclusion: Performing surgery using an artificial implant within 2 weeks of the injury showed better outcomes and fewer postoperative complications than when treatment was delayed.

Abbreviation: CT, computed tomography.

Keywords: Data analysis / Dissection / Orbital fractures / Time-to-treatment

\section{INTRODUCTION}

In orbital floor fractures, it is generally accepted that perform-

\section{Correspondence: Yong Jig Lee}

Department of Plastic and Reconstructive Surgery, Daegu Catholic University

School of Medicine, 33 Duryugongwon-ro 17-gil, Nam-gu, Daegu 42472, Korea

E-mail: syjlee@cu.ac.kr

This work was supported by a grant of the Research Institute of Medical Science, Daegu Catholic University (grant No. 2019-08, 2019).

Received November 10, 2020 / Revised June 11, 2021 / Accepted June 20, 2021 ing surgery within 2 weeks after the injury yields the best outcomes [1]. However, patients are sometimes reluctant to undergo surgical treatment-and particularly to give informed consent regarding the risk of some postoperative complicationsbecause they do not immediately perceive their appearance as having changed after injury. Instead, it is common for patients to request surgical repair around 2 or more weeks after the injury, after their severe edema begins to subside and they start to recognize that their appearance has changed. 
To identify the optimal timing of surgery, excluding cases of emergent exploratory operations for release of potentially trapped extraocular muscles, this retrospective study compared the outcomes of patients with orbital fractures who underwent surgical repair by stratifying them based on the time interval between the injury and surgery.

\section{METHODS}

Patients' charts and computed tomography (CT) images from July 2009 to June 2020 were retrospectively reviewed. Data on age, the timing of surgery, dimensions of the defect, operation time, and follow-up period were collected from plastic surgeons' and ophthalmologists' medical records. Patients who underwent emergency operations for release of muscle entrapment were excluded from the analyses, as were patients who underwent simultaneous treatment for medial wall fractures. The remaining patients were divided into three groups according to the time interval from the injury to surgery (group 1: 3-7 days, group 2: 8-14 days, and group 3: 15 or more days).

This study was approved by the bioethics committee of our institution following a standard institutional review board protocol (IRB No. CR-20-060-L).

The timing of the surgery and decision to perform orbital floor repair was determined by the presence of unchanged diplopia (with or without uncomfortable movement of the extraocular muscle) and sunken eye or enophthalmos. Follow-up at the plastic surgery clinic lasted until patients agreed that their infraorbital paresthesia had recovered and/or subjective diplopia had improved. The author classified the surgical outcomes using a 4 -point scale: $4=\operatorname{good}$ (no complications), $3=$ fair (no subjective symptoms), $2=$ poor (remaining paresthesia), and $1=$ very poor (with strabismus and/or enophthalmos). Patients' participation in decision-making regarding health care and treatment is known to reduce the frequency of elective surgery without any apparent adverse effects on health outcomes or satisfaction [2]. After considering their medical conditions (Table 1) and receiving an explanation from the physician detailing possible postoperative complications and potential changes in symptoms based on initial CT studies and the patients' early symptoms, the patients or their guardians decided between sur- gical treatment or further observation.

\section{Surgical procedure}

A transconjunctival surgical approach was primarily used, except in cases where the transverse dimension of the lesion on the preoperative CT scan was over $2 \mathrm{~cm}$, in which case the subciliary surgical approach was employed using $\times 2.5$ surgical loupes and a surgical scalpel blade \#15. During dissection, a subperiosteal approach was used in procedures performed within 14 days after the injury, and supraperiosteal access was also used in procedures performed at a longer post-injury interval. Bipolar electric cautery was used to perform dissection and ensure bleeding control, and monopolar cautery was used to control bone bleeding after the fracture site was exposed. To release the trapped soft tissue on the floor of the orbit, an ultrasonic piezoelectric instrument (Surgybone; Silfradent, Sofia, Italy) was used to shave the bone. A Medpor and titanium mesh (1-mm Medpor Titan surgical implant; Stryker, Kalamazoo, MI, USA) was used if the defect area was larger or equal to 2 $\mathrm{cm}^{2}$; otherwise, a porous polyethylene implant (Medpor surgical implant, Stryker) without titanium mesh was inserted. The material was fixed with one 4-mm self-drilling screw (MatrixNEURO; DePuy Synthes, Switzerland) or using the tongue-ingroove method. The forced duction test was performed after implant placement to ensure eye mobility [3]. Negative pressure drainage systems were not applied, although they are known to be effective for retrobulbar hemorrhage prevention [4]. In the absence of contraindications, an intravenous injection of 62.5 mg of methylprednisolone was administered once preoperatively and once daily for 2 days postoperatively to control the swelling [5]. The patients usually remained admitted to the hospital for 4-7 days after surgery to receive intravenous antibiotics. Within 4-5 days, the skin sutures (6-0 nylon) were removed in the subciliary approach. In the conjunctival approach, buried sutures were done with 7-0 coated Vicryl (Ethicon, Rari$\tan , \mathrm{NJ}, \mathrm{USA})$.

\section{Evaluation of skin paresthesia on the face}

The author determined the patients' pinprick threshold by assessing their response to the prick of a 23-gauge needle mounted on a $2-\mathrm{mL}$ syringe by gently placing the needle on an area

Table 1. Conditions due to which patients decide on surgery at 15-93 days after injury (group 3)

\begin{tabular}{|c|c|c|}
\hline Conditions & No. of patients $(\%)(n=28)$ & Severity score \\
\hline Multiple trauma (intracranial hemorrhage, subdural hemorrhage, subarachnoid hemorrahge, lung contusion, basal skull fracture) & $8(28.57)$ & 4 \\
\hline Aspirin medication with myocardial infarction intervention & $1(3.57)$ & 3 \\
\hline Minor previous surgery (skin suture, canaliculi repair, etc.) & $3(10.71)$ & 2 \\
\hline No symptoms until the patients recognized sunken eye & $16(57.14)$ & 1 \\
\hline
\end{tabular}


innervated by the infraorbital nerve on the non-injured side of the face and then on the injured side [6]. The responses on both sides of the face were compared. Paresthesia was considered present if patients felt a sharp prick in the non-injured area and experienced a different sensation on the injured area (such as no/blunt pain [a score of 0 ], tingling, or a painful sensation [a score of 10]) on the skin surface of paresthesia.

\section{Evaluation of accompanying conditions}

The author classified the accompanying medical conditions using a 4-point severity score: 4 , multiple trauma; 3 , taking medications that needed to be stopped before surgery; 2 , minor previous surgery; and 1, no symptoms until the patients recognized sunken eye (Table 1).

\section{Statistical analysis}

Data were analyzed using descriptive statistics, the chi-square test, the Kruskal-Wallis H test, Spearman correlation, and binary logistic regression test. For binary logistic regression analysis, the four outcome variable scores were coded as 1 (good) and 2 (fair, poor, and very poor). The statistical analysis was performed using SPSS version 28 (IBM Corp., Armonk, NY, USA). A $p$-value of $<0.05$ was considered to indicate statistical significance.

\section{RESULTS}

In total, 85 patients underwent surgery for inferior orbital wall fractures. Group 1 comprised 25 patients who underwent surgery 3-7 days after the injury, group 2 contained 32 patients with a time to treatment of 8-14 days, and group 3 included 28 patients with an interval of 15-93 days between the injury and surgery. The overall score distribution of the surgical outcomes was as follows: good, 63; fair, 7; poor, 6; and very poor, 9. In groups 1,2 , and 3, the percentage of patients with good outcomes was $84 \%, 81.25 \%$, and $57.14 \%$, respectively (Table 2 ).

The three groups showed no statistically significant differences in the transverse dimension of the injury (group 1: 14.45 \pm 4.40 $\mathrm{mm}$, group 2: $17.07 \pm 5.31 \mathrm{~mm}$, and group 3: $16.50 \pm 4.10 \mathrm{~mm}$; $p=0.110$ ) or the anteroposterior dimension (group 1: 17.37 \pm $6.36 \mathrm{~mm}$, group 2: $18.35 \pm 6.57 \mathrm{~mm}$, and group 3: $20.72 \pm 6.64 \mathrm{~mm}$; $p=0.144$ ). However, the sunken depth of the soft tissue was smaller in group 1 than in group 3 (group 1: $6.12 \pm 2.28 \mathrm{~mm}$ [range, 2.33-11.04 mm], group 2: 7.79 $\pm 4.53 \mathrm{~mm}$ [range, 1.84$23.75 \mathrm{~mm}$ ], and group 3: $8.72 \pm 3.00 \mathrm{~mm}$ [range, 3.16-15.92 $\mathrm{mm}] ; p=0.110)$. The mean operation durations were $171.23 \pm 117.81$ minutes, $167.55 \pm 125.77$ minutes, and 138.48 \pm 118.85 minutes for groups 1,2 , and 3 , respectively $(p=0.625)$. The follow-up periods for paresthesia assessment were, respectively, $12.15 \pm 12.28$ weeks, $9.84 \pm 11.25$ weeks, and $27.38 \pm 30.44$ weeks in groups 1,2 , and 3 , respectively $(p=0.064)$. The followup periods for ophthalmologic examinations to assess diplopia were $13.14 \pm 27.77$ weeks, $10.37 \pm 12.95$ weeks, and $31.67 \pm 48.52$ weeks in the three groups, respectively $(p=0.498)$. The postoperative outcome scores of the three groups were: $3.84 \pm 0.37$ (group 1 ), $3.63 \pm 0.87$ (group 2), and $2.93 \pm 1.33$ (group 3) ( $p=0.083$ ). Descriptive statistics were shown in Table 2.

Transverse dimension of the lesion and the postoperative outcome score showed a weak negative correlation $(\rho=-0.380$, $p=0.004$ ), indicating the larger transverse impact was associated with lower postoperative scores in groups 1 and 2. In group 3, no other transverse, anteroposterior, or craniocaudal dimensional

Table 2. Descriptive statistics of variables $(n=85)$

\begin{tabular}{|c|c|c|c|c|}
\hline Variable & & No. $(\%)$ & Mean \pm SD & Median (IQR) \\
\hline \multirow[t]{4}{*}{ Postoperative outcome score } & 1 (very poor) & $9(10.59)$ & & \\
\hline & 2 (poor) & $6(7.06)$ & & \\
\hline & 3 (fair) & $7(8.24)$ & & \\
\hline & 4 (good) & $63(74.12)$ & & \\
\hline \multirow[t]{2}{*}{ Postoperative outcome score code ${ }^{a)}$} & 1 (good) & $63(74.12)$ & & \\
\hline & 2 (fair, poor, very poor) & $22(25.88)$ & & \\
\hline \multirow[t]{3}{*}{ Group (timing of surgery after injury) } & 1 (3-7 day) & $25(29.41)$ & & \\
\hline & 2 (8-14 day) & $32(37.65)$ & & \\
\hline & 3 (15-93 day) & $28(31.76)$ & & \\
\hline Transverse dimension (mm) & & & $16.11 \pm 4.75$ & $16.57(12.55-18.95)$ \\
\hline Anteroposterior dimension (mm) & & & $18.84 \pm 6.60$ & $18.55(13.82-23.06)$ \\
\hline Sunken depth (mm) & & & $7.60 \pm 3.61$ & $7.43(4.85-9.56)$ \\
\hline Operation time (min) & & & $221.93 \pm 66.31$ & $217.00(165.00-271.50)$ \\
\hline
\end{tabular}

$\mathrm{IQR}$, interquartile range.

a) This outcome score code divided the 4 postoperative outcome scores into 1 and 2 for binary logistic regression analysis. 
Table 3. Correlations between the dimensions of the defects and other variables in Spearman correlation

\begin{tabular}{|c|c|c|c|c|c|c|c|c|}
\hline Group & & $\begin{array}{c}\text { Transverse } \\
\text { dimension (mm) }\end{array}$ & $\begin{array}{l}\text { Anteroposterior } \\
\text { dimension (mm) }\end{array}$ & $\begin{array}{c}\text { Sunken } \\
\text { depth (mm) }\end{array}$ & $\begin{array}{l}\text { Interval between } \\
\text { injury and } \\
\text { operation (day) }\end{array}$ & $\begin{array}{l}\text { Operation time } \\
\text { (min) }\end{array}$ & $\begin{array}{l}\text { Postoperative } \\
\text { outcome scores }\end{array}$ & Severity score \\
\hline Total $(n=85)$ & & & & & & & & - \\
\hline \multirow[t]{2}{*}{ Transverse dimension (mm) } & $\rho$ & 1.000 & $0.514^{\mathrm{a})}$ & $0.458^{\mathrm{a})}$ & 0.123 & 0.198 & -0.199 & \\
\hline & $p$-value & & $<0.001$ & $<0.001$ & 0.263 & 0.069 & 0.068 & \\
\hline \multirow[t]{2}{*}{ Anteroposterior dimension (mm) } & $\rho$ & $0.514^{\mathrm{a})}$ & 1.000 & $0.457^{\mathrm{a})}$ & $0.246^{b)}$ & 0.073 & 0.031 & \\
\hline & $p$-value & $<0.001$ & & $<0.001$ & 0.023 & 0.509 & 0.775 & \\
\hline \multirow[t]{2}{*}{ Sunken depth (mm) } & $\rho$ & $0.458^{\mathrm{a})}$ & $0.457^{\mathrm{a})}$ & 1.000 & $0.294^{\mathrm{a})}$ & -0.074 & -0.144 & \\
\hline & $p$-value & $<0.001$ & $<0.001$ & & 0.006 & 0.502 & 0.189 & \\
\hline Group $1 \& 2(n=57)$ & & & & & & & & - \\
\hline \multirow[t]{2}{*}{ Transverse dimension (mm) } & $\rho$ & 1.000 & $0.424^{\mathrm{a})}$ & $0.478^{a)}$ & 0.208 & 0.198 & $-0.380^{\mathrm{a})}$ & \\
\hline & $p$-value & & 0.001 & $<0.001$ & 0.120 & 0.140 & 0.004 & \\
\hline \multirow[t]{2}{*}{ Anteroposterior dimension (mm) } & $\rho$ & $0.424^{\mathrm{a})}$ & 1.000 & $0.463^{\mathrm{a})}$ & 0.201 & 0.009 & 0.058 & \\
\hline & $p$ value & 0.001 & & $<0.001$ & 0.135 & 0.950 & 0.666 & \\
\hline \multirow[t]{2}{*}{ Sunken depth (mm) } & $\rho$ & $0.478^{a)}$ & $0.463^{\mathrm{a})}$ & 1.000 & 0.122 & -0.009 & -0.142 & \\
\hline & $p$-value & $<0.001$ & $<0.001$ & & 0.366 & 0.948 & 0.292 & \\
\hline \multicolumn{9}{|l|}{ Group $3(n=28)$} \\
\hline \multirow[t]{2}{*}{ Transverse dimension (mm) } & $\rho$ & 1.000 & $0.745^{a)}$ & $0.430^{\mathrm{b})}$ & -0.160 & 0.187 & 0.014 & 0.170 \\
\hline & $p$-value & & $<0.001$ & 0.022 & 0.416 & 0.446 & 0.945 & 0.387 \\
\hline \multirow[t]{2}{*}{ Anteroposterior dimension (mm) } & $\rho$ & $0.745^{\mathrm{a})}$ & 1.000 & $0.447^{b)}$ & -0.135 & 0.222 & 0.204 & 0.235 \\
\hline & $p$-value & $<0.001$ & & 0.017 & 0.492 & 0.256 & 0.299 & 0.229 \\
\hline \multirow[t]{2}{*}{ Sunken depth (mm) } & $\rho$ & $0.430^{b)}$ & $0.447^{b)}$ & 1.000 & -0.106 & -0.129 & 0.095 & -0.238 \\
\hline & $p$-value & 0.022 & 0.017 & & 0.592 & 0.512 & 0.630 & 0.223 \\
\hline \multirow[t]{2}{*}{ Severity score } & $\rho$ & 0.170 & 0.235 & -0.238 & 0.091 & 0.153 & 0.250 & 1.000 \\
\hline & $p$-value & 0.387 & 0.229 & 0.223 & 0.648 & 0.437 & 0.200 & \\
\hline
\end{tabular}

$\rho$, correlation coefficient.

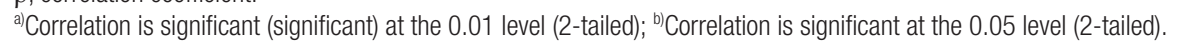

Table 4. Unadjusted and adjusted odds ratios for postoperative outcome code from binary logistic regression $(\mathrm{n}=85)$

\begin{tabular}{lcccc}
\hline Factor & Unadjusted OR & $95 \% \mathrm{Cl}$ & Adjusted OR & $95 \% \mathrm{Cl}$ \\
\hline Timing of surgery (groups 1, 2, and 3) & 2.13 & $1.09-4.18$ & 2.58 & $1.04-6.34$ \\
Transverse dimension (mm) & 1.06 & $0.95-1.17$ & 1.05 & $0.92-1.19$ \\
Anteroposterior dimension (mm) & 1.00 & $0.93-1.07$ & 0.95 & $0.86-1.04$ \\
Sunken depth (mm) & 1.12 & $0.98-1.28$ & 1.08 & $0.92-1.26$ \\
Operation time (min) & 1.00 & $1.00-1.01$ & 0.99 & $0.99-1.01$ \\
\hline
\end{tabular}

$\mathrm{OR}$, odds ratio; $\mathrm{Cl}$, confidence interval.

This outcome score codes: 1 (good in the postoperative outcome score) and 2 (fair, poor, and very poor in the postoperative outcome score).

variables were correlated with the postoperative outcome score. The severity score of the accompanying conditions was also not correlated with any other variables (Table 3 ).

In the binary logistic regression analysis among the timing of surgery, the three dimensions of injury site, and the operation time for the postoperative outcome score codes, it showed that when the group number (timing of surgery) increased, the odds of postoperative outcome scores codes increased 2.58 times $(p=0.041)$ (Table 4$)$.

\section{DISCUSSION}

The craniocaudal dimension or sunken depth of soft tissue her- niation is an accurate and reliable measure that distinguishes patients with acute blowout fractures who require earlier surgical interventions from those who do not [7]. However, in this study, if the volume of soft tissue measured on the initial CT studies could not definitively indicate whether surgical management was necessary, the decision of whether to perform surgery was made based on an evaluation of the reduction in swelling. If patients did not present any noteworthy symptoms (such as sunken eye and/or double vision) over time, they usually did not want to undergo surgery. The physician recommended surgical repair within 2 weeks of the injury if the defect was large, and most patients with large defects therefore agreed to undergo surgery within 2 weeks. The patients observed the 
course of their condition while they noticed attenuation of double vision, and their periorbital appearance became similar to that before the injury. Patients decided to undergo surgical repair if they stopped experiencing improvements in double vision or if they noticed other changes in their periorbital appearance (e.g., sunken eye).

Radiographically, bony union was noted 4 weeks postoperatively. At 8 weeks, the bony defects were completely remodeled, and no radiolucent areas could be seen [8]. If patients preferred for spontaneous bone union to take place without surgical repair, the patients were instructed to avoid putting pressure on the orbital space for 4 weeks and blowing their noses until 12 weeks after the injury, considering the thin weak inferior or medial orbital wall.

Since no significant differences were found among the three groups defined in terms of time to treatment in the transverse $(p=0.110)$ and anteroposterior $(p=0.144)$ dimensions of the defect on preoperative CT, and the choice of implant materials was based on the area of the bone defect, it appeared that the type of implant did not affect the postoperative outcomes. However, the sunken depth of the soft tissue was smaller in group 1 than in group $3(6.12 \pm 2.28 \mathrm{~mm}$ vs. $8.72 \pm 3.00 \mathrm{~mm})$. The patients selected the timing of treatment based on the severity and duration of swelling or discomfort at the injury site. More severe swelling was generally associated with a decision to undergo surgery later. Thus, the sunken depth of soft tissue on the initial CT might predict the duration of swelling.

At 2 weeks after the injury, a cartilaginous matrix, vascular invasion, and osteoblast activity were noted at both the fracture and the fracture ostectomy sites [8]. Subsequently, intraoperative difficulties were expected in dissection due to adhesions between the orbital soft tissue and the bony structure of the fracture site. In group 3, there were adhesions between the orbital soft tissues and the bony fracture edges due to the ruptured fascial system. For reconstructive surgery that uses artificial materials, it is necessary to consider the size of the defect (transverse, and anteroposterior dimensions) to gauge the range of dissection required to separate the herniated soft tissue from the surrounding bony structure. A subperiosteal approach is possible only before the soft tissue and bone healing process begins. Thus, after approximately 14 days, supraperiosteal access was considered necessary, especially around or on the herniated components. There were no statistically significant differences in the operation running time according to the interval until surgery $(p=0.625)$.

Repair of the orbital floor is indicated for large fractures with enophthalmos and if there is clinical evidence of entrapment with positive forced duction and diplopia. Typically, surgery should be delayed for 1-2 weeks after the fracture to allow the orbital and eyelid edema to improve. This permits a better ocular examination and allows for spontaneous improvement in cases where diplopia is due to muscle trauma, hemorrhage, or edema rather than entrapment [9]. Three complications (diplopia, enophthalmos, and hypoesthesia of the infraorbital nerve territory) are still frequently observed at long-term follow-up visits, despite a correct surgical technique and successful anatomic reconstruction [10]. Ocular motility imbalance and resultant diplopia can persist after surgical repair in 10\%-30\% of patients with blowout fractures, and it is reasonable to assume that ocular motility restrictions after orbital floor fractures result from contusion injuries, laceration, avulsion, or hemorrhage in orbital nerves or muscles [11]. Preoperative diplopia is likely to be the most important prognostic factor for predicting postoperative diplopia after surgical repair, but diplopia in blowout fractures without restricted ocular motility could simply be due to edema and observation is recommended as long as it improves [12,13].

To overcome the potential subjective bias of this study, medical records from the ophthalmology department were reviewed thoroughly, and it was found that most patients continued to visit the ophthalmology department for a long time due to eye symptoms even though the follow-up visits at the plastic surgery department to check for paresthesia or enophthalmos had ended much earlier. It is suggested that plastic surgeons and ophthalmologists could meaningfully collaborate in the evaluation and management of the tissues surrounding these injuries, including the reconstruction of bone structures. In this study, $18.82 \%$ of patients showed persistent diplopia, including nine asymptomatic patients with less than $5^{\circ}$ of diplopia.

Based on theories of buckling and hydraulic mechanisms [14], it has been assumed that when higher-energy collisions more strongly affect periorbital or retrobulbar soft tissues than most of the force is transmitted to the underlying bone during the collision, the soft tissues got more swollen and showed slower recovery. Patients with less craniocaudal displacement on the initial CT examination (groups 1 and 2) showed improvements in double vision and decreased swelling. When these improvements ceased, surgical exploration and treatment were carried out within 14 days. However, patients with a larger sunken depth on the initial CT examination (group 3) showed slow improvements in double vision and decreased swelling. When these changes stopped, a decision was made to perform surgical treatment after 14 days. Intraoperatively, the sunken depth was often found to be greater in group 3 than what was expected from the initial CT findings. With the bone fragments that moved downwards together, the periosteum stuck to the bone 
and the neighboring periorbital fascia was also displaced downwards. This may be why the patients in group 3 continued to feel discomfort when moving their extraocular muscles, and the persistent double vision following the injury may have been partly due to herniation of orbital fat and connective tissue into the surrounding sinus with subsequent traction on the muscle sheaths [15]. This could be a possible explanation for why there was no correlation between the sunken depth (craniocaudal dimension) shown on the initial CT examination and patients' postoperative outcome score in group 3 , even though groups 1 and 2 showed negative correlations (Table 3 ).

In conclusion, better outcomes of orbital floor fracture repair with implants seemed to be achieved in group 1 than in group 3 (Table 4). The absence of sunken eye around 2 weeks after the injury due to remaining swelling may not indicate spontaneous healing without any complications; instead, this observation may suggest a poorer prognosis with complications such as diplopia. One goal of orbital reconstruction is to restore ocular motility $[16,17]$. The data on dimensional information from the initial CT is thought to be valid within 2 weeks. Subsequently, an additional preoperative CT re-evaluation is recommended for the surgeon and the patients to have an accurate understanding of the periorbital conditions more than 2 weeks post-injury.

\section{NOTES}

\section{Conflict of interest}

No potential conflict of interest relevant to this article was reported.

\section{Ethical approval}

The study was approved by the Institutional Review Board of Daegu Catholic University Medical Center (IRB No. CR-20060-L) and performed in accordance with the principles of the Declaration of Helsinki. The informed consent was waived.

\section{ORCID}

Yong Jig Lee

https://orcid.org/0000-0002-6470-5750

\section{REFERENCES}

1. Aldekhayel S, Aljaaly H, Fouda-Neel O, Shararah AW, Zaid WS, Gilardino M. Evolving trends in the management of orbital floor fractures. J Craniofac Surg 2014;25:258-61.

2. Stacey D, Legare F, Lewis K, Barry MJ, Bennett CL, Eden KB, et al. Decision aids for people facing health treatment or screening decisions. Cochrane Database Syst Rev 2017;4:CD001431.
3. Kim HS, Jeong EC. Orbital floor fracture. Arch Craniofac Surg 2016;17:111-8.

4. Lee DW, Kim TH, Choi HJ, Wee SY. Delayed-type retrobulbar hematoma caused by low temperature after reconstruction of inferior blow-out fracture. Arch Craniofac Surg 2021;22:110-4.

5. Aldhabaan SA, Hudise JY, Obeid AA. A meta-analysis of preand postoperative corticosteroids for reducing the complications following facial reconstructive and aesthetic surgery. Braz J Otorhinolaryngol 2020 Jun 20 [Epub]. https://doi.org/10.1016/j. bjorl.2020.05.015.

6. Bennett M. The LANSS Pain Scale: the Leeds assessment of neuropathic symptoms and signs. Pain 2001;92:147-57.

7. Mansour TN, Rudolph M, Brown D, Mansour N, Taheri MR. Orbital blowout fractures: a novel CT measurement that can predict the likelihood of surgical management. Am J Emerg Med 2017;35:112-6.

8. Rever LJ, Manson PN, Randolph MA, Yaremchuk MJ, Weiland A, Siegel JH. The healing of facial bone fractures by the process of secondary union. Plast Reconstr Surg 1991;87:451-8.

9. Silbert DI, Matta NS, Singman EL. Diplopia secondary to orbital surgery. Am Orthopt J 2012;62:22-8.

10. Brucoli M, Arcuri F, Cavenaghi R, Benech A. Analysis of complications after surgical repair of orbital fractures. J Craniofac Surg 2011;22:1387-90.

11. Ramphul A, Hoffman G. Does preoperative diplopia determine the incidence of postoperative diplopia after repair of orbital floor fracture? An institutional review. J Oral Maxillofac Surg 2017;75:565-75.

12. Tahiri Y, Lee J, Tahiri M, Sinno H, Williams BH, Lessard L, et al. Preoperative diplopia: the most important prognostic factor for diplopia after surgical repair of pure orbital blowout fracture. J Craniofac Surg 2010;21:1038-41.

13. Alinasab B, Borstedt KJ, Rudstrom R, Ryott M, Qureshi AR, Beckman MO, et al. New algorithm for the management of orbital blowout fracture based on prospective study. Craniomaxillofac Trauma Reconstr 2018;11:285-95.

14. Waterhouse N, Lyne J, Urdang M, Garey L. An investigation into the mechanism of orbital blowout fractures. Br J Plast Surg 1999;52:607-12.

15. Koornneef L. Orbital septa: anatomy and function. Ophthalmology 1979;86:876-80.

16. Jung JS, Kang DH, Lim NK, Kim H. Orbital wall restoration with primary bone fragments in complex orbital fractures: a preliminary study. Arch Craniofac Surg 2020;21:156-60.

17. Kim T, Kim BK. Endoscopic slide-in orbital wall reconstruction for isolated medial blowout fractures. Arch Craniofac Surg 2020;21:345-50. 